\title{
Autogenous rib grafts for reconstruction of the manubrium after resection: Technical refinements and outcomes
}

\author{
Guofei Zhang, MD, Chengxiao Liang, MM, Gang Shen, MD, Wenshan Li, MD, Lijian Huang, MD,
} Saibo Pan, MD, and Ying Chai, MD

\begin{abstract}
Objective: Sternal reconstruction after resection is essential for restoring the rigidity of the chest cavity. However, no consensus has been reached regarding the most physiologic or efficacious material for this procedure. We present our clinical experience with autogenous rib grafts for reconstruction of the manubrium after resection and discuss the refinements in this procedure.
\end{abstract}

\begin{abstract}
Methods: Twelve consecutive patients with a confirmed diagnosis of a primary neoplasm in the manubrium between March 2003 and August 2013 were analyzed retrospectively. All patients underwent manubrium resection and immediate reconstruction with autogenous rib grafts.
\end{abstract}

\begin{abstract}
Results: No cases of perioperative mortality were noted. The median operation time was 129 minutes. One patient experienced mild paradoxical movement; the other patients recovered well without any complications. The median follow-up period after surgery was 69 months. Two patients died. One patient with chondrosarcoma died as a result of cardiac disease 26 months after surgery and another patient with non-Hodgkin lymphoma died as a result of local recurrence 6 months after surgery. Computed tomographic scans for the other 10 patients have shown neither dislocation nor abnormality of the transplanted ribs; moreover, no absorption of the grafts or recurrence was noted. None of the patients required prescription analgesics for the pain at the donor site at the 3-month follow-up visit.
\end{abstract}

Conclusions: This case series demonstrates the successful use of autogenous rib grafts in the reconstruction of the manubrium after resection. We consider that the reconstruction technique is a safe and effective alternative to a complex problem. (J Thorac Cardiovasc Surg 2014;148:2667-72)

Primary and secondary sternal tumors are uncommon. In addition, benign lesions in the sternum are very rare; most sternal tumors are malignant, and usually include sarcomas that originate from either the bone or the soft tissues of the sternum. ${ }^{1,2}$ Although the role of surgical treatment in such cases remains controversial, it should be considered the best option in most patients with primary and secondary malignant tumors involving the sternum, and it can achieve a definitive or palliative cure. ${ }^{3}$ However, resection of the entire sternum or part of the sternum without any reconstruction may result in paradoxical movement of the thorax and impaired ventilation. Moreover, reconstruction of bony and soft tissue defects of the sternum after resection can also restore chest wall rigidity, which helps to protect the underlying mediastinal structures.

The material used for reconstruction of the sternum has evolved considerably over the last several decades. ${ }^{4,5}$

\footnotetext{
From the Department of Thoracic Surgery, the Second Affiliated Hospital, College of Medicine, Zhejiang University, Hangzhou, China.

Disclosures: Authors have nothing to disclose with regard to commercial support.

Guofei Zhang and Chengxiao Liang are co-first authors.

Received for publication Jan 10, 2014; revisions received April 25, 2014; accepted for publication July 9, 2014; available ahead of print Sept 5, 2014.

Address for reprints: Ying Chai, MD, Department of Thoracic Surgery, the Second

Affiliated Hospital, College of Medicine, Zhejiang University, No. 88 Jiefang

Rd, Hangzhou, 310009 China (E-mail: zetszju@yahoo.com).

$0022-5223 / \$ 36.00$

Copyright (c) 2014 by The American Association for Thoracic Surgery

http://dx.doi.org/10.1016/j.jtcvs.2014.07.093
}

Generally, the requirements of an ideal material for use in the reconstruction of the sternum include availability, durability, nonreactivity, and resistance to infection. Although different materials have been described for sternal reconstruction, including autograft, allograft, nondegradable and biodegradable synthetic materials, and tissue engineering products, there is no statistical evidence, either from retrospective comparative series or from prospective randomized trials, to show a significant difference in outcome. , $^{4,7}$

The manubrium is a flat bone; it is slightly convex anteriorly and concave posteriorly. The upper border of this area includes the jugular notch and the upper lateral borders include the clavicular notches, which join with the clavicles and receive the attachments of the sternocleidomastoid muscles. ${ }^{8}$ Because of the anatomical characteristics of the manubrium, its resection and reconstruction present special difficulties. In our initial report on the use of autogenous rib grafts for the reconstruction of a middle segment defect of the sternum, we proposed that autogenous rib grafts offered important advantages over other materials. ${ }^{9}$ We have subsequently used this technique in the resection and reconstruction of the manubrium. In the present study, we aimed to retrospectively review our experience of autogenous rib grafts for reconstruction of the manubrium after resections in 12 patients during a 10-year period. 


\section{Abbreviations and Acronyms}

$\mathrm{CT}=$ computed tomography

MRI $=$ magnetic resonance imaging

$\mathrm{PTFE}=$ polytetrafluoroethylene

\section{MATERIALS AND METHODS}

\section{Study Population}

Before the study, the Research Review Board at our institution examined and approved our research protocol, which was in accordance with the Declaration of Helsinki. All patients provided written informed consent before surgery.

Between March 2003 and August 2013, 12 consecutive patients with a confirmed diagnosis of a primary neoplasm in the manubrium underwent resection and reconstruction using autogenous rib grafts. All patients were evaluated preoperatively using chest radiography, computed tomography (CT), magnetic resonance imaging (MRI), or a combination of these (Figure 1). The precise location of the tumor, the extent of sternal involvement and any infiltration into the adjacent structures were assessed to determine whether it was possible to perform an en bloc radical resection.

Of the 12 patients included in the study, 5 were men and 7 were women; the mean ( \pm standard deviation) age was $47.7 \pm 12.4$ years (range, 23-68 years). The chief complaints before surgery were the presence of a palpable sternal mass in 7 patients, pain in 3, and a mass with pain in 2. Four patients underwent preoperative fine-needle aspiration of the lesion and the result clearly indicated the presence of a primary malignant sternal tumor. Three patients underwent positron emission tomography-CT scanning to confirm that the disease was localized to the sternum with no distant metastases. The patients' clinical characteristics are presented in Table 1.

\section{Operative Technique}

The patient is intubated using a double-lumen tube under general anesthesia and is then placed in the supine position. The surgical procedure comprises 3 steps as described in the following.

Manubrium resection. The first step involved skin excision. When the skin and overlying soft tissues are not involved, a vertical elliptical incision is made, encompassing the aspiration site. The skin excision must be large in cases of ulceration, or if the tumor involves subcutaneous tissues. The extent of sternal resection depends on the nature of the tumor and the extent of sternum involvement. First, we determine whether the sternoclavicular joint or the sternal extremity of the clavicle is involved. If these are not involved, we resect the manubrium along the surface of the sternoclavicular joint. However, if the sternoclavicular joint or the sternal extremity of the clavicle is involved, the sternoclavicular joint is divided with a Gigli saw at the normal part of the clavicle, after dissection of the surrounding ligament tissue. It is important to avoid injuring the subclavian vessels and nerve during dissection. The tumors are generally resected $3 \mathrm{~cm}$ beyond the margin of the lesions, and may include any costal cartilages involved. In all cases, the resection margins are simultaneously examined by the frozen section procedure. The sternum is usually divided with a Gigli saw (Figure 2). We avoid entering the pleural space; however, if the tumor extends into the chest cavity, the extent of the tumor in the upper or lower mediastinum is then assessed. In these cases, the lung and mediastinal structures involved need to be excised en bloc. After sternal resection, a chest tube is placed in the pleural cavity if the pleural space has been entered.

Rib harvesting technique. A left or right anterior lateral incision is made parallel to the underlying rib that is to be harvested. The incision depth is increased through the subcutaneous tissue, and passes through muscular tissue and fascia. The muscle is separated in the direction of its fibers. Rib grafts are harvested from the subperiosteum, leaving the periosteum and pleural portions intact (Figure 3). The ribs are removed using a
Gigli saw and infiltrated into a saline solution. When possible, the remaining rib periosteum is closed to a tubular form by a separate layer using a 20 absorbable suture. The skin is then closed in layers.

Sternal reconstruction. The reconstruction must afford sufficient stability and adequate airtight closure. The ribs are carved into 2 or 3 clips using a Gigli saw as needed. The ends of the 2 rib clips, clavicle, and the edge of the sternum are perforated using an electric drill. The rib clips are placed on the edge of the sternum and clavicle using rib nails, and then fixed using stainless steel wire. The rib grafts are placed to form a $\mathrm{T}, \mathrm{V}$, or D shape between the edges of the residual sternum, costal cartilage, and clavicle (Figure 4). For patients with an intact sternoclavicular joint and a small defect after resection, the $\mathrm{V}$ position is chosen; however, for patients in whom the sternoclavicular joint and/or partial clavicle are involved and resected, the $\mathrm{T}$ or $\mathrm{D}$ position is used. The main aim is to restore the stability of the clavicle, and hence, the $\mathrm{T}$ position is used for a small defect and the D position is used for a large defect. Thereafter, a drainage tube is placed below the transplanted ribs to drain the effusion after the grafting procedure. Parts of the pectoralis major muscles are then mobilized to cover the grafts. The skin is closed then in layers.

\section{Follow-up and Data Collection}

The patients were followed up at 4 weeks, 3 months, 6 months, and 1 year postoperatively. Follow-up data were obtained from subsequent clinic visits and examinations and telephone interviews with patients and families. All patients were evaluated for pain at the donor sites. Routine chest CT scans were obtained at every follow-up visit to exclude dislocation or abnormality of the transplanted ribs, absorption of the grafts, and recurrence.

The continuous variables are presented as the mean \pm standard deviation. All statistical analyses were performed using SPSS for Windows, version 16 (SPSS, Inc, Chicago, Ill).

\section{RESULTS}

All patients underwent a radical en bloc resection of the tumor and involved structures and reconstruction with autogenous rib grafts. Five patients underwent total resection of the manubrium, 2 underwent resection of the manubrium and partial resection of the sternal body, 3 underwent simultaneous partial costal cartilage resection, and 2 underwent simultaneous partial lung resection. The median operation time was 129 minutes (range, 90-170 minutes) and the median blood loss was $140 \mathrm{~mL}$. Only 1 patient required a blood transfusion. No cases of perioperative mortality were noted. All the patients were extubated within 24 hours of the operation. One patient experienced mild paradoxical movement after this reconstruction; this was because only rib nails were used without fixing with stainless steel wire, which resulted in slippage of the rib grafts. The other patients recovered well without any respiratory complications, wound infection or dehiscence, or instability of the sternum. For all patients, the mean postoperative hospital stay was 7.4 days (range, 5-11 days).

On microscopic examination, all margins were tumor free in all patients. The most common malignant histologic type was chondrosarcomas ( 5 cases); the other primary tumors included 1 desmoid tumor, 1 non-Hodgkin lymphoma, 1 plasmacytoma, and 1 malignant fibrous histiocytoma. Three patients had benign tumors, which included 


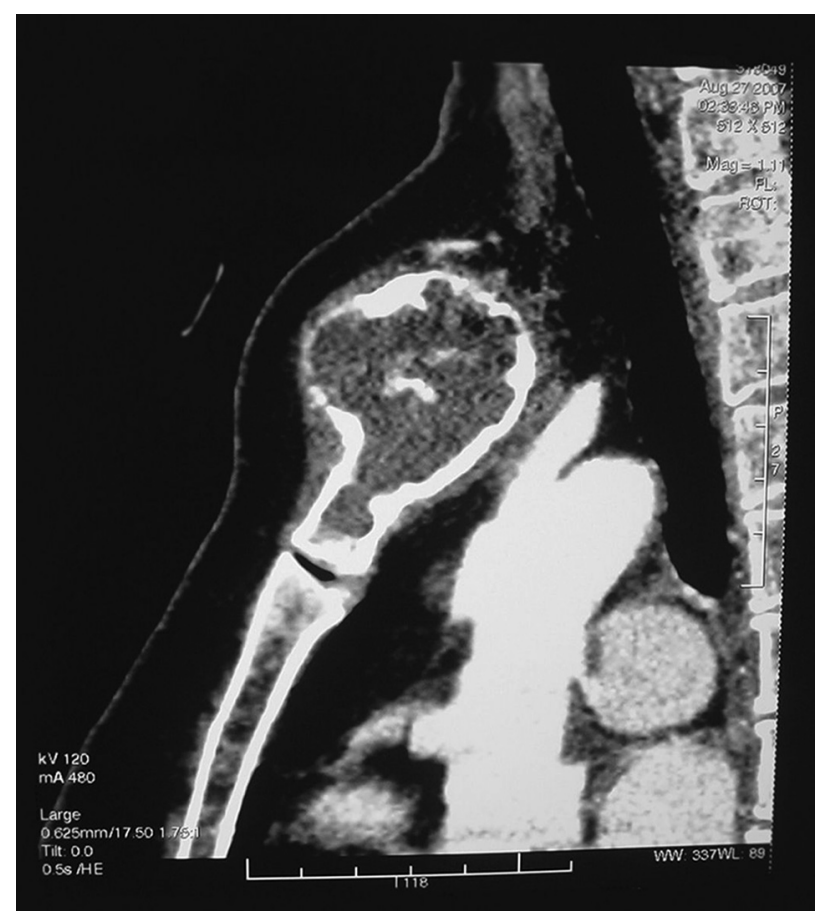

FIGURE 1. Computed tomographic scan showing massive invasion in the manubrium.

chondroma, chronic suppurative osteomyelitis, and fibrous dysplasia.

Postoperative adjuvant chemotherapy and radiotherapy were administered after sternectomy in 2 patients (1with non-Hodgkin lymphoma and 1 with plasmacytoma).

The patients were observed until September 2013. The median follow-up period after surgery was 69 months (mean, $64.2 \pm 38.9$ months), and 2 patients died during this period. One patient with chondrosarcoma died of cardiac disease 26 months after surgery. In the other patient who had been diagnosed with non-Hodgkin lymphoma, local recurrence was observed, despite postoperative adjuvant chemotherapy; this patient died 6 months after surgery. Annual follow-up for the other 10 patients is ongoing. CT scans of the reconstructed chest have shown neither dislocation nor abnormality of the transplanted ribs, and there has been no absorption of the grafts or recurrence. None of the patients required prescription analgesics for the pain at the manubrium graft site at their 3-month follow-up visit.

\section{DISCUSSION}

Surgical treatment should be considered the best option in most patients with primary and secondary malignant tumors involving the sternum, and it can achieve a definitive or palliative cure. ${ }^{3,10}$ However, sternal reconstruction after resection is essential to prevent flap movement and consequent paradoxical respiration, and to restore chest wall rigidity to protect the underlying mediastinal structures. $^{4,11,12}$ The material used for sternal reconstructions has evolved considerably over the last several decades. ${ }^{4,5}$ However, there is no statistical evidence, either from retrospective comparative series or from prospective randomized trials, to show a significant difference in outcome. In this case series, we retrospectively reviewed our experience of using autogenous rib grafts for the reconstruction of the manubrium after resection in 12 patients. We believe that this technique is a safe and efficient approach with good results.

The primary aim of resection for a sternal malignant tumor is to achieve complete (R0) resection. Most benign tumors can be adequately detected on plain films; however, CT or MRI is necessary to precisely define the anatomical location of lesions and plan the extent of resection. For malignant sternal tumors, previous studies recommended en bloc resection, covering an area extending at least $3 \mathrm{~cm}$ beyond the margin of the tumor, and including structures such as the lungs, thymus, and pericardial tissues that may be invaded by the tumor. ${ }^{6}$

TABLE 1. Clinical characteristics of the 12 patients who underwent manubrium resection and reconstruction

\begin{tabular}{|c|c|c|c|c|c|c|}
\hline Case & $\operatorname{Age}(y) / \operatorname{sex}$ & Symptoms & Tumor size (cm) & Histologic type & Postoperative treatment & Survival (mo) \\
\hline 1 & $42 / \mathrm{F}$ & $\mathrm{P}$ & $8 \times 7$ & Chondrosarcoma & - & 68/D \\
\hline 2 & $52 / \mathrm{F}$ & M & $6 \times 5$ & Fibrous dysplasia & - & $116 / \mathrm{A}$ \\
\hline 3 & $23 / \mathrm{M}$ & $P$ & $4 \times 4$ & Chondroma & - & $111 / \mathrm{A}$ \\
\hline 4 & $39 / \mathrm{F}$ & $\mathrm{M}, \mathrm{P}$ & $3 \times 4$ & Malignant fibrous histiocytoma & - & $104 / \mathrm{A}$ \\
\hline 5 & $55 / \mathrm{M}$ & M & $4 \times 5$ & Chronic suppurative osteomyelitis & - & $102 / \mathrm{A}$ \\
\hline 6 & $68 / \mathrm{F}$ & $\mathrm{M}, \mathrm{P}$ & $4 \times 4$ & Chondrosarcoma & - & 71/A \\
\hline 7 & $53 / \mathrm{F}$ & M, P & $5 \times 5.5$ & Chondrosarcoma & - & 70/A \\
\hline 8 & $38 / \mathrm{M}$ & M & $5 \times 5$ & Non-Hodgkin lymphoma & $\mathrm{CT}+\mathrm{RT}$ & $15 / \mathrm{D}$ \\
\hline 9 & $55 / \mathrm{F}$ & $\mathrm{M}, \mathrm{P}$ & $8 \times 7$ & Chondrosarcoma & - & $49 / \mathrm{A}$ \\
\hline 10 & $41 / \mathrm{M}$ & M & $3.5 \times 4$ & Chondrosarcoma & - & $4 / \mathrm{A}$ \\
\hline 11 & $63 / \mathrm{M}$ & M & $4 \times 4$ & Desmoid tumor & - & $32 / \mathrm{A}$ \\
\hline 12 & $43 / \mathrm{F}$ & M & $4 \times 4$ & Plasmacytoma & $\mathrm{CT}+\mathrm{RT}$ & 28/A \\
\hline
\end{tabular}

$F$, Female; $M$, male; $M$, mass; $P$, pain; $C T$, chemotherapy; $R T$, radiation therapy; $D$, dead; $A$, alive. 


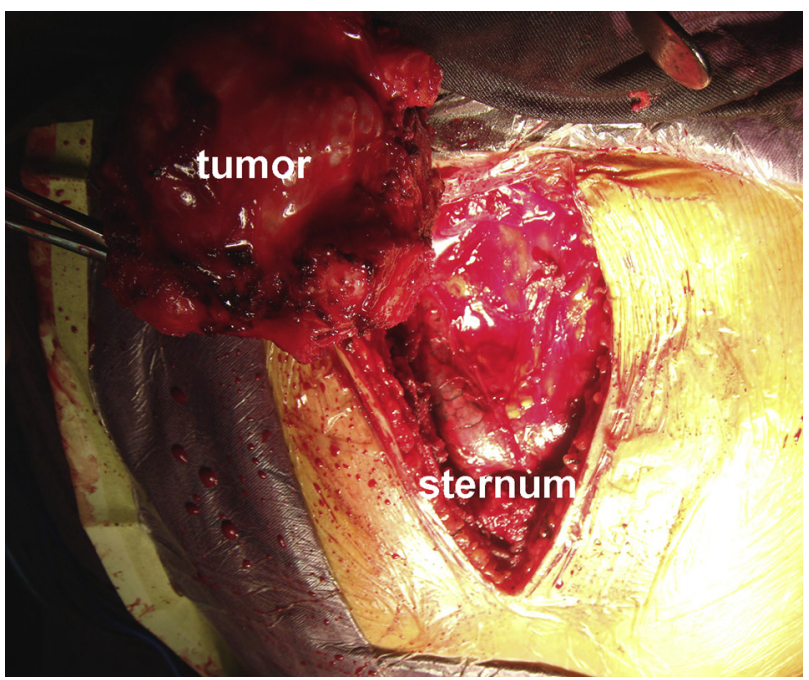

FIGURE 2. Chondrosarcoma of the manubrium after resection.

In general, prosthetic materials for reconstruction should achieve the objective function without inducing a foreign body response, facilitate smooth wound healing, and resist infection. ${ }^{13}$ However, no consensus has been reached regarding the most physiologic or efficacious material to be used in such procedures. Therefore, the choice of prosthesis, including synthetic materials and autogenous tissues, is based mainly on the surgeon's preference. At present, the synthetic materials used in sternal reconstruction include polypropylene/high-density polyethylene mesh, polytetrafluoroethylene (PTFE), polypropylene/PTFE mesh, vicryl nets, polytetrafluoroethylene patch, methylmethacrylate, stainless steel mesh, resin plate, or metal plate. ${ }^{4,6,7,14}$ Although these synthetic materials are commercially available, with guaranteed source and quality, diversity, durability, and internal inertness, these reconstruction materials are not completely devoid of complications and are generally removed if wound infection, symptoms of mediastinitis, sepsis, pyothorax, or intolerance to medical treatment develop in the acute or chronic phase.

The use of autogenous tissues for sternal reconstruction has been proposed. Because of its rapid incorporation, decreased immune response, more robust vascularization, and accelerated osteointegration, the iliac crest is a popular site for obtaining autologous bone grafts. ${ }^{15,16}$ However, a few studies have reported on cases of donor site morbidity with the use of the iliac crest graft. Silber and colleagues ${ }^{17}$ reported the results of a retrospective questionnaire involving 134 patients who received an iliac crest autograft and underwent an average follow-up of 4 years. The acute postoperative complications included difficulty with ambulation $(51 \%)$, extended antibiotic usage $(7.5 \%)$, and persistent drainage $(3.7 \%)$. The chronic symptoms included pain at the donor site in $26.1 \%$ of patients, rated at a mean visual analog scale score of 3.8 out of 10 . Therefore, autogenous



FIGURE 3. Rib grafts harvested from the subperiosteum, leaving the periosteum and pleural portions intact.

rib grafting, which has been proved suitable for reconstruction in maxillofacial surgery, seems to be a good option. ${ }^{18-20}$ In our initial report on autogenous rib grafts for reconstruction of the middle segment defect of the sternum, we maintained that our rib grafts offered important advantages over other materials, such as rigidity to eliminate the possibility of paradoxical chest movement, inertness to reduce the risk of infection associated with prosthetic materials, and radiolucency to facilitate radiographic inspection. Moreover, they offered good incorporation into the tissues and could easily be fixed under tension to the edges of the sternal defect. In addition, rib grafts are easy to obtain and have a lower financial cost for patients compared with prosthetic materials.

Certain details of our technique should be highlighted. We always choose the seventh to ninth rib for reconstruction, as these ribs can be obtained easily through relatively short incisions. After the ribs are identified, they are carefully dissected to leave their periosteum and pleural portions intact. Because the muscle fibers and the periosteum are left intact, patients experience little postoperative pain. Moreover, because of their different characteristics, we excise the rib and retain the costal cartilage. To achieve the best possible connection at the interface between the rib grafts and the sternum, we use a Gigli saw to transect the ribs, clavicle, and manubrium. Drainage tubes can be placed around the transplanted ribs to drain the effusion.

The prerequisites for ensuring the successful use of autogenous rib grafts for reconstruction of the sternum include (1) good condition of the recipient site (ie, a good blood supply to the skin and subcutaneous tissue and fresh margins without abnormal bony tissue or infected tissue); 


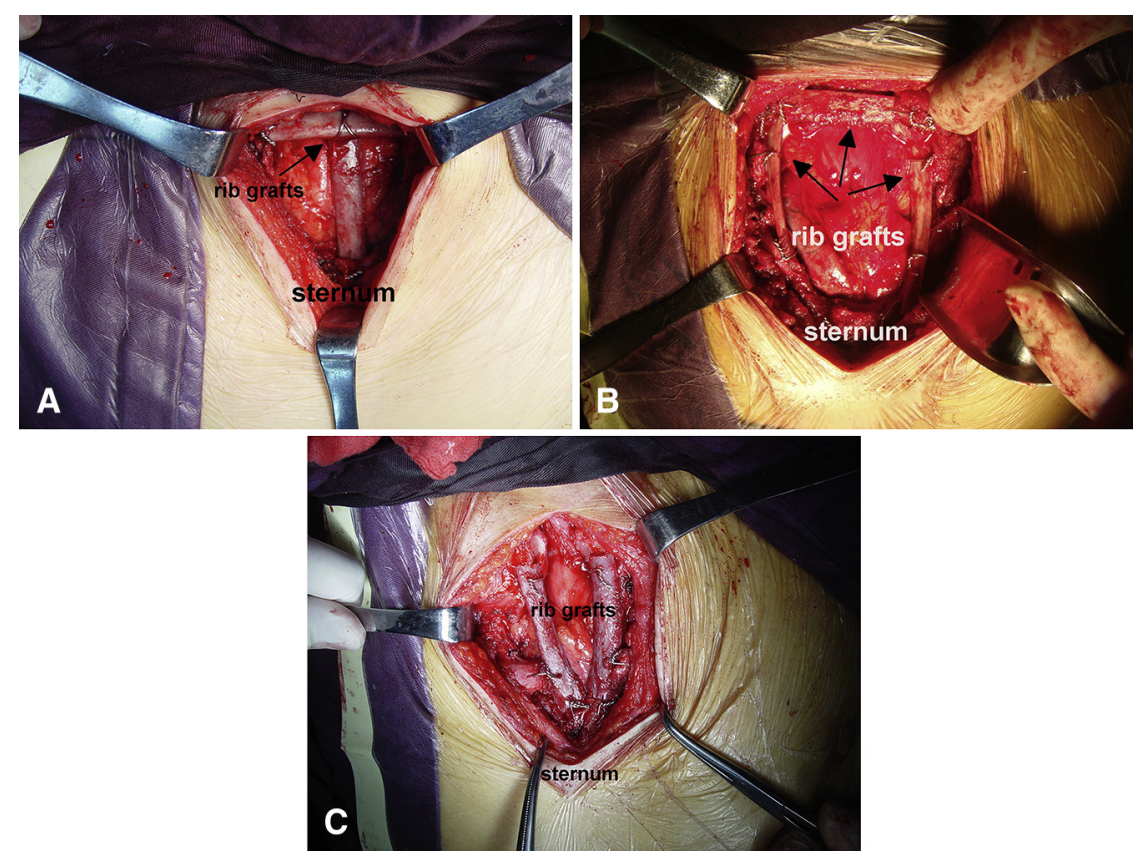

FIGURE 4. Reconstruction with autogenous rib grafts. The rib grafts were placed in the form of a $\mathrm{T}(\mathrm{A}), \mathrm{V}(\mathrm{B})$, or $\mathrm{D}$ (C) between the edges of the residual sternum, costal cartilage, and clavicle, where they were fixed using stainless steel wire.

(2) sufficient stability of the contact surface of the bones; and (3) the shortest possible exposure of the grafts in vitro (because the surviving osteoblasts on the surface of the bone grafts can produce new bony tissue).

When rib graft harvests are considered, most surgeons typically have concerns about chest complications. ${ }^{21,22}$ Skouteris and colleagues ${ }^{23}$ reported a retrospective study evaluating 28 patients in whom a total of 31 rib resections had been performed. They noted a pleural tear in 1 patient during the harvesting of a costochondral rib graft, which was repaired without any resultant pneumothorax. No incidence of pneumothorax, postoperative pulmonary complications, or wound infection or breakdown was reported. Long-term follow-up showed that none of the patients experienced any chronic donor site discomfort, and all patients accepted the appearance of their scars. In our retrospective case series, the technique has been found to be safe and efficient. There were no cases of perioperative mortality. One patient experienced mild paradoxical movement with this reconstruction, as only rib nails were used without fixation with stainless steel wire; the other patients recovered well without any complications. Thus far, none of the patients have reported pain at the donor site from the time of hospital discharge to follow-up. Apart from 1 case with nonHodgkin lymphoma, no radiological artifact or scattering was observed during the oncological follow-up with both $\mathrm{CT}$ and MRI.

\section{Limitations}

There are several limitations to our study. First, compared with procedures involving other synthetic materials, our procedures require an additional incision for rib harvesting; however, no complications at the rib resection site were observed during the follow-up. Second, this study was retrospective and lacked a control group; therefore, the results of our approach for reconstruction could not be compared with those of other prosthetic materials. A prospective randomized study is needed to compare our approach more precisely with other approaches. Third, in our case series, only 4 patients underwent preoperative fine-needle aspiration of the lesion. We recommend that all patients with a sternal tumor should undergo a preoperative biopsy or fine-needle aspiration of the lesion to clarify the pathology and thus avoid unnecessary surgery. Although some details of the reconstruction were described, the size of the cohort was relatively small. Therefore, further studies with larger patient populations will be needed to verify the favorable outcomes of manubrium reconstruction using this technique.

\section{CONCLUSIONS}

In our series of patients, autogenous rib grafts were successfully used for reconstruction of the manubrium after resection. We consider that the reconstruction technique using autogenous rib grafts is safe and effective, and offers a simple and satisfactory solution to a complex problem.

\section{References}

1. Chapelier A. Resection and reconstruction for primary sternal tumors. Thorac Surg Clin. 2010;20:529-34

2. Incarbone M, Nava M, Lequaglie C, Ravasi G, Pastorino U. Sternal resection for primary or secondary tumors. J Thorac Cardiovasc Surg. 1997;114:93-9. 
3. Galbis Caravajal JM, Sánchez LY, Fuster Diana CA, Jorge RG, Ortiz PF, Deaville PJ. Sternal resection and reconstruction after malignant tumours. Clin Transl Oncol. 2009;11:91-5.

4. Aghajanzadeh M, Alavy A, Taskindost M, Pourrasouly Z, Aghajanzadeh G, Massahnia S. Results of chest wall resection and reconstruction in 162 patients with benign and malignant chest wall disease. J Thorac Dis. 2010;2:81-5.

5. Haraguchi S, Hioki M, Hisayoshi T, Yamashita K, Yamashita Y, Kawamura J, et al. Resection of sternal tumors and reconstruction of the thorax: a review of 15 patients. Surg Today. 2006;36:225-9.

6. Liu H-s, Qin Y-z, Li S-q, Li L, Cui Y-s, Zhang Z-y. Surgical resection of sternal tumors and reconstruction with titanium mesh. Chin Med Sci J. 2011;26:237-40.

7. Fabre D, El Batti S, Singhal S, Mercier O, Mussot S, Fadel E, et al. A paradigm shift for sternal reconstruction using a novel titanium rib bridge system following oncological resections. Eur J Cardiothorac Surg. 2012;42:965-70.

8. Xiu P, Shui D, Wang Q, Wang G, Lan Y. Anatomic and morphometric analysis of manubrium sterni as a source of autograft for anterior cervical fusion surgery using quantitative 3-dimensional computed tomographic scans. Spine (Phila Pa 1976). 2012;37:E935-41.

9. Chai Y, Zhang G, Shen G. Autogenous rib grafts for reconstruction of sternal defects after partial resection: a new surgical technique. Plast Reconstr Surg. 2008; 121:353e-5e.

10. Mansour KA, Anderson TM, Hester TR. Sternal resection and reconstruction. Ann Thorac Surg. 1993;55:838-42; discussion 43.

11. Pyle JW, Ash JL, Hussain SM, Reid N, Anderson RC. Sternal resection and reconstruction after renal cell carcinoma metastatic to the sternum. J Thorac Cardiovasc Surg. 2005;129:1177-8.

12. Lequaglie C, Massone PB, Giudice G, Conti B. Gold standard for sternectomies and plastic reconstructions after resections for primary or secondary sternal neoplasms. Ann Surg Oncol. 2002;9:472-9.
13. Soysal O, Walsh GL, Nesbitt JC, McMurtrey MJ, Roth JA, Putnam JB Jr. Resection of sternal tumors: extent, reconstruction, and survival. Ann Thorac Surg. 1995;60:1353-8; discussion 8-9.

14. Nishiyamaki N, Inoue K, Nakatani S, Takeuchi S, Hamba H, Kawaguchi T, et al. Chest wall resection in general thoracic surgery. Osaka City Med J. 2002;48: 77-84.

15. Ren P, Zhang J, Zhang X. Resection of primary sternal osteosarcoma and reconstruction with homologous iliac bone: case report. J Formos Med Assoc. 2010; 109:309-14.

16. Bapat MR, Chaudhary K, Garg H, Laheri V. Reconstruction of large iliac crest defects after graft harvest using autogenous rib graft: a prospective controlled study. Spine (Phila Pa 1976). 2008:33:2570-5.

17. Silber JS, Anderson DG, Daffner SD, Brislin BT, Leland JM, Hilibrand AS, et al. Donor site morbidity after anterior iliac crest bone harvest for single-level anterior cervical discectomy and fusion. Spine (Phila Pa 1976). 2003;28:134-9.

18. Wulkan M, Sa AJ, Alonso N. Modified technique to increase nostril crosssectional area after using rib and septal cartilage graft over alar nasal cartilages. Acta Cir Bras. 2012;27:713-9.

19. Christophel JJ, Hilger PA. Osseocartilaginous rib graft rhinoplasty: a stable, predictable technique for major dorsal reconstruction. Arch Facial Plast Surg. 2011; 13:78-83.

20. Toriumi DM. Autogenous grafts are worth the extra time. Arch Otolaryngol Head Neck Surg. 2000;126:562-4.

21. Moretti A, Sciuto S. Rib grafts in septorhinoplasty. Acta Otorhinolaryngol Ital. 2013;33:190-5.

22. Kaji N, Asato H. Reconstruction of the auricle with autogenous rib cartilage grafts. Adv Otorhinolaryngol. 2014;75:61-75.

23. Skouteris CA, Sotereanos GC. Donor site morbidity following harvesting of autogenous rib grafts. J Oral Maxillofac Surg. 1989;47:808-12. 